Anaesthesist 2009 · 58:339-340

DOI 10.1007/s00101-009-1555-7

Online publiziert: 8. April 2009

(c) Springer Medizin Verlag 2009

V. Wenzel ${ }^{1}$ B. Zwißler ${ }^{2} \cdot$ R. Larsen ${ }^{3}$

${ }^{1}$ Univ.-Klinik für Anaesthesie und Intensivmedizin, Medizinische Universität Innsbruck, Innsbruck

${ }^{2}$ Klinik für Anästhesiologie der LMU, Klinikum Großhadern, München

${ }^{3}$ Klinik für Anästhesiologie, Universitätskliniken, Homburg/Saar

\title{
Manipulierende Wissenschaftler
}

- ein klinischer Effekt von NSAID und COX-2-Hemmern auf eine Knochenheilung unklar ist und

- multimodale präemptive Analgetikastrategien zur Verhinderung von chronischen Schmerzen nach großen orthopädischen Eingriffen nicht bewiesen sind [13].

Interessanterweise haben die meisten der jetzt zurückgezogenen Studien von Dr. Reuben neue Analgetika oder neue Applikationswege untersucht; diese wurden jeweils als günstig beschrieben.

Wie kann es passieren, dass Koautoren, Gutachter und Herausgeber diese Manipulationen nicht bemerkt haben? Es ist vermeintlich einfach zu erklären: Wissenschaft basiert auf Vertrauen. Wenn Daten von einem geschickten Fälscher plausibel manipuliert werden, ist eine Entdeckung sehr unwahrscheinlich. Dies zeigt uns, dass wir vielleicht misstrauischer sein müssten. Im Rahmen der Qualitätskontrolle der Manuskriptgutachten bei Annals of Emergency Medicine wurde festgestellt, dass zwei Drittel der Gutachter schwere Mängel [z. B. Randomisierung durch Münzwurf um Mitternacht oder visuelle Analogskala (VAS) sechsstatt zehnstufig; [2]] in einem Testartikel nicht gefunden hatten. Auch 10 Jahre später fanden weniger als $40 \%$ der Gutachter vom British Medical Journal in einen Testartikel eingebaute Fehler (z. B. Randomisierung nach Familiennamen; [9]). Als einen Ansatz zur Lösung bei der Aufarbeitung einer Datenmanipulation in der
Innsbrucker Universitätsklinik für Urologie [11] nannten die Herausgeber von The Lancet: „... wissenschaftliche Anerkennung setzt Verantwortung der Koautoren voraus" [8]. Wenn das unmittelbare Umfeld eines manipulierenden Wissenschaftlers keine Auffälligkeiten bemerkt oder solche nicht beanstandet, wird die Wahrscheinlichkeit der anschließenden Entdeckung durch Gutachter, Herausgeber und Leser einer Fachzeitschrift immer geringer. Wird unprofessionelles Verhalten geduldet, sind mehr unprofessionelle Vorgänge wahrscheinlich [7]. Umso schwerer sind dann der Schaden und der Vertrauensverlust auch für unbeteiligte Wissenschaftler, Universitäten, Fachzeitschriften, Patienten und Stipendiengeber, wenn Missstände nicht verhindert, sondern sogar in Fachzeitschriften [1] und in der Laienpresse diskutiert werden $[3,4,6]$. Wir alle müssen daher in unserem beruflichen Umfeld, z. B. bei der Qualitätskontrolle von Studien oder der Entscheidung über Autorenschaften, nicht nur Professionalität und Integrität vorleben [12], sondern diese auch unmissverständlich und von allen an unserer Arbeit Beteiligten einfordern, um fundamentale Probleme zu verhindern [5].

\section{Korrespondenzadresse \\ Prof. Dr. V. Wenzel M.Sc. M.Sc. \\ Univ.-Klinik für Anaesthesie \\ und Intensivmedizin, \\ Medizinische Universität Innsbruck \\ Anichstr. 35, 6020 Innsbruck \\ Österreich \\ volker.wenzel@uki.at}


Interessenkonflikt. Der Autor hat keinen Interessenskonflikt in Bezug auf die in diesem Artikel diskutierten Inhalte.

\section{Literatur}

1. Abbott A (2008) Report finds grave flaws in urology trial. Nature 454:922

2. Baxt WG, Waeckerle JF, Berlin JA, Callaham ML (1998) Who reviews the reviewers? Feasibility of using a fictitious manuscript to evaluate peer reviewer performance. Ann Emerg Med 32:310-317

3. Berndt C (2008) Eine österreichische Lösung. Süddeutsche Zeitung, 02.09.2008

4. Charisius H (2009) Schmerzfreier Fälscher. Namhafter US-Anästhesist erfand 13 Jahre lang Studien. Süddeutsche Zeitung, 24.03.2009, S 1

5. Epstein RM, Hundert EM (2002) Defining and assessing professional competence. JAMA 287:226235

6. Harris $G$ (2009) Doctor admits pain studies were frauds, hospital says. New York Times, 11.03.2009, SA22

7. Hickson GB, Pichert JW, Webb LE, Gabbe SG (2007) A complementary approach to promoting professionalism: identifying, measuring and addressing unprofessional behaviors. Acad Med 82:10401048

8. Kleinert S, Horton R (2008) Retraction - autologous myoblasts and fibroblasts versus collagen corrected for treatment of stress urinary incontinence in women: a corrected randomised controlled trial. Lancet 372:789-790

9. Schroter S, Black N, Evans S et al (2008) What errors do peer reviewers detect and does training improve their ability to detect them? J R Soc Med 101:507-514

10. Shafer S (2009) Tattered threads. Anesth Analg (in press)

11. Strasser H, Marksteiner R, Margreiter E et al (2007) Autologous myoblasts and fibroblasts versus collagen for treatment of stress urinary incontinence in women: a randomised controlled trial. Lancet 369:2179-2186

12. Wenzel V, Dunser MW, Lindner KH (2007) How do I write an original article? An introduction for beginners. Anaesthesist 56:828-836

13. White PF, Kehlet $H$, Liu S (2009) Perioperative analgesia - what do we still know? Anesth Analg (in press)

\section{Schwenk, Spies, Müller Fast Track in der operativen Medizin}

Heidelberg: Springer-Verlag 2009, 786 S., (ISBN 978-3-540-39708-3), gebunden, 89.95 EUR

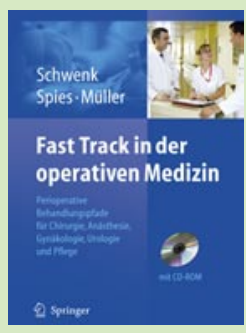

Der Chirurg versteht oft unter "fast-track" in erster Linie „wenig invasive Zugänge" und "schnelle Entlassung", der Anästhesist versteht unter fast-track oft "thorakale Epiduralanästhesie" und "schnelle Entlassung", der Krankenhausökonom oft nur "schnelle Entlassung". Dabei vereint Fast track surgery verschiedene Techniken, die in der perioperativen Patientenbehandlung nach elektiven Operationen angewandt werden. Das aktuelle Verständnis der perioperativen Pathophysiologie hat gezeigt, dass multiple Faktoren zur postoperativen Morbidität, Verkürzung der Krankenhausbehandlung und beschleunigten Erholung beitragen. Die Verbesserungen des chirurgischen Ergebnisses verlangen nach multidisziplinären Ansätzen, die bisher erfolgreich v.a. bei Kolonresektion eingesetzt wurden [gerade das Berliner interdisziplinäre Herausgebertrio aus Anästhesie und Chirurgie war in Deutschland maßgeblicher Motor]. Dabei wurde zunächst oft verkannt, dass dieses Behandlungskonzept zwanglos auf viele operative Eingriffe von der Hysterektomie oder Prostatektomie über Hüft und Knieendoprothetik bis zur Aortenchirurgie und Carotisdesobliteration übertragen werden kann, wie dieses Buch überzeugend vermittelt. Die vorgestellten Techniken umfassen u.a. minimal invasive Techniken und eine optimale Schmerztherapie insbesondere mittels Epidural- oder Regionalanästhesie, kurzwirksame, gut steuerbare Anästhetika mit wenig emetogener Wirkung sowie eine postoperative Rehabilitation mit früher enteraler Ernährung und Mobilisierung. Die Kombination dieser Herangehensweisen reduziert die perioperative Stressantwort und Organdysfunktion und verkürzt daher deutlich die Zeit zur vollen Rehabilitation.

Häufig wird "fast-track" dabei in erster Linie unter ökonomischen Gesichtspunkten ge- sehen (und kritisiert:„,blutige Entlassung“). Dieser Vorwurf wird durch die Autoren entkräftet: Fast-track spiegelt den ureigensten Patientenwunsch wieder (,Wann kann ich wieder nach Hause") und führt nachweislich zu niedrigeren Komplikationsraten (z.B. Pneumonien).

Die Prozessverdichtung und Spezialisierung führten zu einer immensen Zahl von Schnittstellen in der Medizin, die den Weg zu zielorientierten, multidisziplinären Konzepten wie Fast-track Chirurgie, Clinical Pathways etc. möglich gemacht haben. Wenngleich die heute etablierten Konzepte in Ihrer Struktur noch sehr heterogen sind, so ist allen gemeinsam, Patientenbezogene Endpunkte durch konzertierte und zumeist modulare Programme zu verbessern.

Die Herausgeber und Autoren haben ein bisher fehlendes deutschsprachiges Werk vorgestellt. Die in Checklisten-artiger Darstellung etablierten Konzepte bieten wertvolle Informationen für eine breite Leserschaft: Klinisch tätige Ärzte, klinisch forschende Ärzte, Pflegepersonal, Medizinstudenten, Qualitätsmanager und kaufmännische Entscheidungsträger. Dieses Buch spricht eine potentielle Leserschaft an, die so multidisziplinär sein wird wie die vorgestellten Konzepte. Und so bewirkt "fast-track" noch etwas ganz Besonderes: Es verbessert die Kommunikation nicht nur interdisziplinär zwischen Operateuren und Anästhesisten, sondern auch interprofessionell zwischen Ärzten, Pflegekräften, Physiotherapeuten, Ökotrophologen, Krankenhausmanagern etc.

Kurzum, dieses Buch ist zum einen eine unverzichtbare Fundgrube für Beispiele und Checklisten, wie ein Lexikon gleichermaßen für alle, die fast-track Konzepte in ihrem Verantwortungsbereich implementieren, ausbauen und nachhaltig pflegen wollen.

H. Wulf (Marburg) 